\title{
Evidencias psicométricas de Inventario SISCO SV-21 para el estudio del estrés académico en universitarios peruanos
}

\section{Psychometric evidence of SISCO SV-21 Inventory for the study of academic stress in Peruvian university students}

\author{
Lincol Orlando Olivas-Ugarte* \\ Universidad César Vallejo, Perú \\ ORCID: https://orcid.org/0000-0001-7781-7105 \\ Shanny Fiorella Morales-Hernández \\ Universidad César Vallejo, Perú \\ ORCID: https://orcid.org/0000-0002-8258-8471 \\ Milagros Kateryn Solano-Jáuregui \\ Universidad César Vallejo, Perú \\ ORCID: https://orcid.org/0000-0001-8430-9117
}

Recibido17-08-20 Revisado 29-06-21 Aceptado 25-07-21 En línea 06-09-21

*Correspondencia

Email: lolivas2021@gmail.com
Citar como:

Olivas-Ugarte, L., Morales-Hernandez, S., \& Solano-Jáuregui, M. (2021). Evidencias psicométricas de Inventario SISCO SV-21 para el estudio del estrés académico en universitarios peruanos. $\begin{array}{llll}\text { Propósitos } \quad y \quad \text { Representaciones, } & 9(2), & 647 .\end{array}$ http://dx.doi.org/10.20511/pyr2021.v9n2.647 


\section{Resumen}

Esta investigación instrumental se realizó con el objetivo de analizar las evidencias psicométricas del inventario SISCO SV-21 en universitarios de Lima Metropolitana. Participaron 560 estudiantes de universidades públicas y privadas, de ambos sexos, entre 18 y 50 años. Se corroboró el ajuste del modelo de tres factores correlacionados mediante un análisis factorial confirmatorio: $\mathrm{CFI}=.929$, TLI $=.920, \mathrm{RMSEA}=.083$ [.078-.088], SRMR=.061. Asimismo, la confiabilidad se estimó con el coeficiente omega $(\omega>.80)$ para los tres factores: estresores $=.90$, síntomas $=.89$, y estrategias de afrontamiento $=89$. Por lo tanto, se concluye que el Inventario SISCO SV-21 reúne evidencias de validez y confiabilidad para su uso en universitarios de Lima.

Palabras clave: estrés académico, estudiantes universitarios, validez, confiabilidad.

\section{Summary}

This instrumental research was carried out with the objective of analyzing the psychometric evidences of the SISCO SV-21 inventory in university students of Metropolitan Lima. 560 students from public and private universities participated, of both sexes, between 18 and 50 years old. The fit of the model of three correlated factors was corroborated by a confirmatory factor analysis: $\mathrm{CFI}=.929, \mathrm{TLI}=.920, \mathrm{RMSEA}=.083$ [.078-.088], $\mathrm{SRMR}=.061$. Likewise, reliability was estimated with the omega coefficient $(\omega>.80)$ for the three factors: stressors $=.90$, symptoms $=.89$, and coping strategies $=.89$. Therefore, it is concluded that the SISCO SV-21 Inventory gathers validity and reliability evidence for its use in university students in Lima.

Keywords: academic stress, university students, validity, reliability.

\section{Introducción}

En los últimos meses los estudiantes universitarios se encuentran realizando sus asignaturas en la modalidad no presencial, debido a las medidas de inmovilidad y aislamiento social que se han tomado por la pandemia debido al nuevo coronavirus. En ese sentido, los resultados de una encuesta dirigida a decanos y rectores de 172 universidades de los Estados Unidos sobre el impacto del Covid-19 durante el periodo de marzo último demostró que el $90 \%$ presentó preocupación continua en la salud y bienestar socioemocional de sus estudiantes, el 76\% presentó preocupación en el acceso a la tecnología y plataforma, mientras que el 75\% demostró preocupación sobre aspectos pedagógicos en la educación online (Organización de las Naciones Unidas para la Educación, la Ciencia y la Cultura [UNESCO], 2020).

En el contexto Latinoamericano, el Ministerio de Educación de Chile (MINEDUC, 2019) realizó una encuesta sobre la salud mental universitaria a través de la Comisión Nacional Científica y Tecnológica (CONCYTEC) aplicando un cuestionario sociodemográfico a 600 estudiantes. Los resultados evidenciaron que, el $46 \%$ presenta sintomatología ansiosa-depresiva, $54 \%$ estrés; $51 \%$ pensamientos suicidas, $87 \%$ malos hábitos alimenticios, $67.2 \%$ problemas de sueño y consumo de sustancias. Otro dato relevante es que el $56 \%$ de los encuestados reportó nunca haber solicitado ayuda psicológica mientras que el $44 \%$ confirmó haber recibido tratamiento psicológico.

Entonces, si bien es cierto que recibir las clases a distancia que brindan actualmente las universidades es un factor estresante para los estudiantes, también es innegable que los estudiantes contribuyen negativamente incrementar sus niveles de estrés con malos hábitos de estudio, de sueño y de alimentación. Al respecto, Barrera (2019) afirma que, la presente generación no necesariamente pide ayuda psicológica, porque muchas veces se asume que hacerlo es un signo 
de debilidad. Cuando mes bien, por el contrario, la comunidad universitaria en su totalidad debe asumir la responsabilidad del desarrollo integral de los estudiantes.

Mientras tanto en el Perú, el Ministerio de Educación implementó lineamientos para el cuidado integral de la salud mental en las universidades. Según el portal digital del estado peruano, 21 universidades encuestadas arrojaron que un $85 \%$ de estudiantes evidenciaron problemas de salud mental, un $82 \%$ ansiedad como caso más recurrente, un $79 \%$ en casos de estrés y un $52 \%$ en violencia académica. Como medida, se sugiere poner énfasis no solo en la educación, sino también en la salud mental de los universitarios, promoviendo el bienestar y la salud (MINEDU, 2019).

También, American Psychological Associaton (APA, 2013) define al estrés como una sensación de agotamiento, confusión e inquietud; que altera a la persona ante cualquier estimulo en su vida sin diferenciar clase económica, edad o sexo acarreando problemas emocionales, mentales y físicos donde la experiencia emocional vivida viene acompañada de cambios conductuales, bioquímicos y fisiológicos. Es necesario precisar que el estrés no es sinónimo de ansiedad. El estrés es una respuesta psicosomática ante estímulos de angustia, mientras que la ansiedad se caracteriza por una gran inquietud que genera conmoción, intranquilidad, nerviosismo y preocupación.

Sin embargo, el estudio del estrés se inició formalmente con Selye (1936), considerado como el padre de este estado, lo define como una respuesta ante rotundos estímulos repetitivos del cuerpo ante estímulos estresores, denominando "Síndrome general de adaptación". Sin embargo, Selye (1973) precisó al estrés como aquella reacción no específica del organismo ante requerimientos que sobre él se realiza". Lo característico del estrés es que se adecúa frente a un escenario y conllevando a una reacción inespecífica. Aunque tres décadas después, Lazarus (1966) definió que, el estrés se origina en relación al sujeto y, el ambiente que evalúa como intimidante y dificultoso hacia el afrontamiento desde el inicio escolar hasta los estudios universitarios generándose sobrecargas académicas. A esto se le designa como estrés en el ámbito académico, dándose de manera individual como grupal.

En el presente siglo, Román, Hernández y Ortiz (2008) señalan varios conflictos en el estudio de estrés académico con respecto a la conceptualización, pues el dilema de este es la corriente personológica presentando limitaciones claras en su adecuada contextualización en el ámbito educativo. Cabe mencionar que, otras complicaciones secundarias son: 1) La imprevisión de las actividades en la educación, 2) Incongruencias teórico-metodológicas entre los enfoques, conceptos y los instrumentos utilizados en los estudios, y 3) El tratamiento exclusivo del estímulo estresor, que debilita y resta confianza en lograr un afrontamiento positivo del estrés.

Berrio y Mazo (2011) definieron al estrés como todo estímulo externo que puede perturbar a la persona: los agentes estresores, precisó también consecuencias físicas y mentales añadiendo el factor de afrontamiento ante el estrés, que se refiere a cómo el sujeto hace frente a la presión de su entorno. Por otro lado, Rossi (2012) describe al estrés como una tensión interna causada por escenarios agobiantes en el cual el hombre se adapta. Sin embargo, cada sujeto de forma independiente, mantiene diferentes niveles de preocupación y ansiedad.

La teoría de valoraciones cognitivas del estrés, formulada por Lazarus (1966), se basa en los aspectos cognoscitivos. Esta teoría forma parte de modelos transaccionales de estrés pues considera que la persona se relaciona en situaciones concretas tomando en cuenta la influencia de sus evaluaciones y cogniciones. Dicha teoría destaca la influencia de dos variables, la primera es cuando la persona evalúa el agente estresor y la segunda es cuando los recursos personales, culturales y sociales orientan al enfrentamiento del individuo y el agente estresor.

Por otro lado, la teoría general de sistemas de Bertalanfy (1976) se basa mediante interacciones, sucesos y condiciones que influyen sobre el comportamiento de la persona a través de un proceso sistémico. La persona toma desde su perspectiva un sistema abierto, esto quiero 
decir que siempre recibirá información del entorno en un circuito continuo de entrada conocido como input (estímulos externos) y salida u output (respuestas del sujeto) de información con el fin de alcanzar un equilibrio sistémico. Esto es un proceso de estímulos y respuesta entre sistemas interactivos (Colle, 2002).

Más específicamente, Orlandini (1996) contextualiza al estrés académico en el ámbito estudiantil, ocasionando en el individuo un proceso de cambios emocionales, físicos y físiológicos por la tensión del entorno académico. Sin embargo, Barraza (2007a) presenta un concepto de estrés académico como el desarrollo psicológico y adaptativo: cuando un estudiante se encuentra sujeto a cargas identificadas como estresores genera un desequilibrio sistémico, presentándose como un conjunto de síntomas, optando por medidas de afrontamiento para regresar a un equilibrio sistémico.

El estrés académico, según Barraza (2018) posee como componentes dimensiones estresores, síntomas-reacciones y por ultimo las estrategias de afrontamiento. La presente investigación considera estos componentes como las dimensiones de la variable, por lo que se detallan a continuación:

Los estresores se impulsan cuando "un estudiante es sometido a sobrecargas de tareas estimuladas y evaluadas por el docente a través de tiempos ilimitados y la poca claridad de información para los trabajos" (p. 31). Preexisten diferentes indicadores que generan el estrés en universitarios tales como: la personalidad y carácter de los docentes, otros indicadores son el nivel de exigencia y forma de evaluar.

Los síntomas se aprecian cuando los estudiantes muestran reacciones físicas y mentales como ansiedad, problemas de concentración, fatiga crónica, depresión, tristeza, angustia, desesperación, sentimientos de agresividad e irritabilidad, tendencia a discutir, y desgano para realizar las actividades académicas (Barraza, 2018).

Por último, el estudiante demuestra la adquisición de estrategias de afrontamiento cuando se enfrenta estímulos estresores. Por ejemplo, al concentrarse en resolver una situación preocupante, analizando lo positivo y negativo, manteniendo un control firme sobre sus emociones y recordando como resolvió otros momentos de estrés para conseguir tranquilizarse (Barraza, 2018).

Entre los instrumentos en lengua española para medir el estrés académico destaca el inventario sistémico cognoscitivista SISCO, elaborado por Barraza (2007b), compuesto originalmente por 31 ítems. Este instrumento ha sido aplicado en diferentes estudios a nivel internacional.

En Chile, Jerez y Oyarzo (2016) encontraron una alta prevalencia del estrés académico en una muestra de 314 estudiantes de la universidad de Los Lagos en Osorno. Se verificó la confiabilidad del inventario $(\alpha>$.70). Los resultados indican que el $98 \%$ de los estudiantes presentaron estrés académico; además, las mujeres (96.24\%) mostraron más estrés que los hombres $(88.57 \%)$. Los estresores más frecuentes fueron las evaluaciones de los profesores $(95.6 \%)$ y la sobrecarga de tareas y trabajos $(92.3 \%)$.

También en Ecuador, Álvarez, Gallegos y Herrera (2018) estudiaron el estrés en 210 alumnos de educación superior pública en Guayaquil. El inventario presento una alta confiabilidad $(\alpha=.894)$ y se concluyó que la sobrecarga de tareas es uno de los principales causantes de estrés.

Finalmente, Castillo et al. (2018), en una muestra de 587 universitarios, 89,6\% mujeres, entre 20 y 52 años, de la cuidad de Bogotá, encontraron que un $90.8 \%$ presentó períodos de ansiedad durante el lapso del semestre, un $48.2 \%$ confirmó haber tenido un exceso de demandas académicas, un $42 \%$ alcanzó un nivel medianamente moderado hacia el estrés y un $41.7 \%$ sostuvo que las destrezas de afrontamiento más manejadas fueron las actividades religiosas. 
A nivel nacional, Ancajima (2017) ejecutó un estudio del inventario SISCO con el fin de determinar sus propiedades psicométricas en una muestra 845 estudiantes universitarios, entre 18 y 25 años, del $1^{\circ}$ y $11^{\circ}$ ciclo en la ciudad de Trujillo. Se evidenció la validez basada en la estructura interna en el AFC (RMSEA=.076, GFI=.82, CFI=.84) siendo ajustes aceptables. Referente a confiabilidad, se obtuvo un estadístico del coeficiente de omega alcanzando un puntaje de .85 en estímulos estresantes, .74 en estrategias de afrontamiento y un .94 en síntomas. Concluyendo la validez y confiabilidad del inventario SISCO para la evaluación del estrés académico.

Más recientemente, Barraza (2018) presentó una versión mejorada del SISCO compuesto por 21 ítems. La fiabilidad del inventario presentó niveles aceptables $(\alpha>.85)$. Asimismo, el análisis factorial exploratorio permitió establecer tres factores que explican el $47 \%$ de la varianza total. Estos factores afectan a los componentes procesuales que propone el modelo sistémico cognoscitivista: 1) Estresores, 2) Síntomas, y 3) Estrategias de afrontamiento.

Este instrumento, denominado SISCO SV-21, asume la teoría sistémico-cognoscitivista presentando procesos de estímulos estresores. En primer lugar, el estudiante se ve sujeto a una serie de demandas bajo la valoración del propio estudiante, considerado también estresores que ingresan como (input) quien ocasiona un desequilibrio sistémico. En segundo lugar, aquellos estresores originan un desequilibrio sistémico con relación estudiante y entorno creando un proceso (coping) finalmente, dicho coping crea un impulso de salida (output) hacia las estrategias de afrontamiento restaurando el equilibrio sistémico (Barraza, 2006, p. 126).

No obstante, el Inventario SISCO SV-21 no cuenta con evidencias psicométricas en universitarios peruanos. En tal sentido, esta investigación tiene como objetivo analizar las evidencias de validez y confiabilidad del SISCO SV-21 en estudiantes universitarios de Lima Metropolitana, lo que permitirá su correcta aplicación en el contexto psicoeducativo y realizar otras investigaciones sobre el estrés académico.

\section{Método}

\section{Diseño y tipo de investigación}

Este es un estudio de diseño instrumental (Ato, López \& Benavente, 2013; Montero \& León, 2002), y específicamente de tipo psicométrico (Cortada de Kohan, 2002; Alarcón, 2013), que tiene como finalidad analizar las evidencias psicométricas preliminares del Inventario SISCO SV21 en una muestra de estudiantes universitarios.

\section{Participantes}

Respondieron el formulario 700 universitarios. No obstante, vez concluida la fase de recolección de información, se depuraron 140 protocolos que no cumplieron con los criterios de exclusión: aquiescencia (tendencia lineal), dobles respuestas, $\mathrm{u}$ omisiones (respuestas en blanco). Por lo tanto, se incluyó en la muestra de estudio a 560 estudiantes, 239 de instituciones públicas y 321 de universidades privadas, 160 hombres y 400 mujeres, de 18 a 50 años $(M=22.7, D E=4.0)$, inscritos en semestre 2020-I, seleccionados mediante un muestreo no probabilístico por conveniencia.

\section{Instrumentos}

El Inventario SISCO SV-21 es un instrumento de autoinforme para uso en el contexto psicoeducativo, que contiene 21 reactivos con formato de respuesta tipo Likert de seis alternativas de respuesta, desde nunca $=0$ hasta siempre $=5$, distribuidos en tres factores: estresores (ítems 17), síntomas (ítems 8-14) y estrategias de afrontamiento (ítems 15-21, redactados en sentido inverso). 


\section{Procedimiento}

Inicialmente, se solicitó el permiso del autor del Inventario SISCO SV-21 mediante una carta a nombre de la Escuela Profesional de Psicología de la Universidad César Vallejo. Una vez obtenida autorización para el uso del instrumento se procedió con la recolección de datos a través de la herramienta formularios de Google, distribuida por redes sociales. Los estudiantes fueron informados sobre el objetivo del estudio; asimismo, se les remarcó que su participación era voluntaria, y que toda la información recabada sería completamente anónima y únicamente con fines de investigación. Todo lo mencionado se plasmó en el consentimiento informado que se entregó junto con los instrumentos como parte del protocolo utilizado en el estudio.

\section{Análisis de datos}

Se utilizó el programa R versión 3.6.2 (R Development Core Team, 2007) y específicamente la librería "lavaan" (Rosseel et al., 2018) para evaluar la estructura interna de la prueba mediante un análisis factorial confirmatorio, introduciendo como insumo la matriz de correlaciones policóricas en combinación con el estimador de mínimos cuadrados ponderados robustos (WLSMV), debido a la naturaleza ordinal de los ítems (Brown, 2015), y porque permite detectar relaciones estructurales con precisión en presencia de leve o moderada asimetría (Li, 2014). Finalmente, se usó el coeficiente omega (McDonald, 1999) para estimar la confiabilidad por el método de consistencia interna, considerando valores $\geq .80$ como aceptables.

\section{Resultados}

\section{Matriz de correlaciones policóricas}

En principio, en la tabla 1 se presenta la matriz de correlaciones policóricas de los ítems del inventario.

\section{Tabla 1}

Correlaciones policóricas de los ítems del inventario SISCO SV-21 (n=560)

\begin{tabular}{|c|c|c|c|c|c|c|c|c|c|c|c|c|c|c|c|c|c|c|c|c|c|}
\hline İtems & $\begin{array}{l}\text { S1 } \\
\end{array}$ & $\begin{array}{l}\text { S2 } \\
\end{array}$ & S3 & $\begin{array}{l}\text { S4 } \\
\end{array}$ & S5 & S6 & S7 & S8 & S9 & S10 & $\begin{array}{l}\text { S11 } \\
\end{array}$ & S12 & S13 & S14 & S15 & S16 & S17 & $\begin{array}{l}\text { S18 } \\
\end{array}$ & S19 & S20 & S21 \\
\hline S1 & - & & & & & & & & & & & & & & & & & & & & \\
\hline S2 & .48 & - & & & & & & & & & & & & & & & & & & & \\
\hline S3 & .57 & .61 & - & & & & & & & & & & & & & & & & & & \\
\hline S4 & .53 & .56 & .68 & - & & & & & & & & & & & & & & & & & \\
\hline S5 & .56 & .52 & .66 & .73 & - & & & & & & & & & & & & & & & & \\
\hline S6 & .63 & .44 & .50 & .52 & .55 & - & & & & & & & & & & & & & & & \\
\hline S7 & .43 & .49 & .51 & .46 & .47 & .48 & - & & & & & & & & & & & & & & \\
\hline S8 & .48 & .36 & .36 & .37 & .44 & .40 & .36 & - & & & & & & & & & & & & & \\
\hline S9 & .44 & .37 & .36 & .36 & .35 & .36 & .41 & .59 & - & & & & & & & & & & & & \\
\hline $\mathrm{S} 10$ & .56 & .37 & .40 & .46 & .43 & .45 & .43 & .61 & .75 & - & & & & & & & & & & & \\
\hline S11 & .37 & .24 & .33 & .31 & .34 & .32 & .35 & .40 & .45 & .49 & - & & & & & & & & & & \\
\hline $\mathrm{S} 12$ & .41 & .28 & .26 & .29 & .36 & .28 & .33 & .45 & .52 & .54 & .47 & - & & & & & & & & & \\
\hline $\mathrm{S} 13$ & .40 & .31 & .31 & .32 & .34 & .25 & .30 & .38 & .47 & .49 & .44 & .75 & - & & & & & & & & \\
\hline $\mathrm{S} 14$ & .45 & .34 & .44 & .42 & .43 & .43 & .38 & .46 & .50 & . 48 & .59 & .46 & .49 & - & & & & & & & \\
\hline S15 & .01 & .03 & -.04 & .00 & -.01 & -.02 & .00 & .04 & .02 & .04 & -.01 & .04 & .01 & .03 & - & & & & & & \\
\hline S16 & .01 & .06 & .05 & .08 & .11 & .05 & .11 & .13 & .14 & .09 & .09 & .10 & .07 & .18 & .62 & - & & & & & \\
\hline S17 & .13 & .06 & .07 & .05 & .09 & .09 & .07 & .17 & .18 & .12 & .14 & .14 & .11 & .19 & .45 & .59 & - & & & & \\
\hline S18 & .23 & .15 & .11 & .13 & .13 & .09 & .13 & .14 & .27 & .26 & .19 & .24 & .25 & .14 & .37 & .50 & .55 & - & & & \\
\hline S19 & .08 & .07 & .06 & .07 & .09 & .13 & .10 & .12 & .15 & .15 & .13 & .08 & .05 & .12 & .31 & .44 & .49 & .53 & - & & \\
\hline S20 & .09 & .04 & .08 & .10 & .12 & .10 & .09 & .13 & .21 & .14 & .17 & .15 & .10 & .24 & .43 & .56 & .50 & .48 & .55 & - & \\
\hline S21 & .14 & .08 & .16 & .17 & .16 & .11 & .11 & .20 & .30 & .23 & .17 & .20 & .18 & .26 & .39 & .52 & .58 & .60 & .54 & .63 & - \\
\hline$M$ & 3.71 & 2.89 & 3.29 & 3.20 & 3.26 & 3.89 & 3.37 & 3.20 & 2.98 & 3.27 & 3.29 & 2.76 & 2.70 & 3.27 & 1.80 & 1.69 & 1.74 & 1.91 & 1.84 & 1.94 & 1.86 \\
\hline$D E$ & 0.81 & 1.12 & 1.04 & 1.10 & 1.07 & .95 & 1.21 & 1.19 & 1.28 & 1.11 & 1.07 & 1.27 & 1.20 & 1.12 & .98 & .97 & 1.12 & 1.02 & 1.05 & 1.20 & 1.13 \\
\hline
\end{tabular}

\section{Evidencias de validez basada en la estructura interna}

Se aplicó un análisis factorial confirmatorio para poner a prueba tres distintos modelos de medida. En la tabla 2 se destaca que el segundo (M2) y el tercer modelo (M3) mostraron buenos índices de ajuste: $\mathrm{CFI}=.929$, TLI $=.920$, RMSEA $=.083$ [.078-.088], SRMR $=.061$; además, son equivalentes. No obstante, se decidió descartar el M3 porque se encontró un caso Heywood entre el factor general de estrés hipotetizado y el factor 2 correspondiente a los síntomas de estrés $(\lambda$ =1.07). Por lo tanto, se mantuvo el modelo propuesto por Barraza (2018). 


\section{Tabla 2}

Índices de ajuste para los modelos del Inventario SISCO SV-21 (n=560)

\begin{tabular}{|c|c|c|c|c|c|c|c|c|c|}
\hline \multirow{2}{*}{ Modelos } & \multirow{2}{*}{$\mathrm{X}^{2}$} & \multirow{2}{*}{$\mathrm{gl}$} & \multirow{2}{*}{$\mathrm{X}^{2} / \mathrm{gl}$} & \multirow{2}{*}{ CFI } & \multirow{2}{*}{ TLI } & \multirow{2}{*}{ RMSEA } & \multicolumn{2}{|c|}{ RMSEA $90 \%$} & \multirow{2}{*}{ SRMF } \\
\hline & & & & & & & Inferior & Superior & \\
\hline M1: Ortogonal & 2617.956 & 189 & 13.85 & .759 & .732 & .152 & .146 & .157 & .197 \\
\hline M2: Oblicuo & 901.805 & 186 & 4.85 & .929 & .920 & .083 & .078 & .088 & .061 \\
\hline M3: Segundo orden & 901.805 & 186 & 4.17 & .929 & .920 & .083 & .078 & .088 & .061 \\
\hline
\end{tabular}

Nota: M1, modelo ortogonal; M2, modelo oblicuo; M3, modelo de segundo orden.

En la tabla 3 se aprecia que todas las cargas factoriales son mayores a .50; sin embargo, las correlaciones del factor 3 con respecto al factor $1(r=.163)$ y al factor $2(r=.278)$ son evidencia de que las puntuaciones del inventario deben interpretarse independientemente, lo que no contradice la teoría del estrés planteada para la elaboración del instrumento.

\section{Tabla 3}

Cargas factoriales y correlaciones interfactoriales del modelo oblicuo $(n=560)$

\begin{tabular}{cccc}
\hline Ítems & F1 & F2 & F3 \\
\hline S1 & .773 & & \\
S2 & .679 & & \\
S3 & .789 & & \\
S4 & .806 & & \\
S5 & .813 & & \\
S6 & .707 & & \\
S7 & .655 & .699 & \\
S8 & & .788 & \\
S9 & & .835 & \\
S10 & & .637 & .7677 \\
S11 & & .738 & .738 \\
S12 & & .720 & .742 \\
S13 & & & .665 \\
S14 & & & .742 \\
S15 & & & \\
S16 & & & \\
S17 & & & \\
S18 & & & \\
S19 & & & \\
S20 & & & \\
S21 & & & \\
\hline F1 & & & \\
F2 & .672 & & \\
F3 & .163 & & \\
\hline
\end{tabular}

\section{Fiabilidad}

Finalmente, se examinaron las evidencias de fiabilidad por el método de consistencia interna con el coeficiente omega $(\omega)$, usando como insumo las cargas factoriales estandarizadas que se reportaron en la tabla 3. En ese sentido, se contraron altos niveles de fiabilidad para cada uno de los tres factores: $\mathrm{F} 1=.90, \mathrm{~F} 2=.89, \mathrm{~F} 3=.89$ (Campo-Arias y Oviedo, 2008; Hogan, 2004; Peters, 2014). 


\section{Discusión}

La presente investigación se ejecutó con la finalidad de analizar las evidencias psicométricas, validez y confiabilidad, del Inventario SISCO SV-21 para el estudio de estrés académico en estudiantes universitarios peruanos, específicamente de Lima Metropolitana, que han desarrollado sus labores académicas en durante el contexto de la emergencia sanitaria causada por el Covid-19.

Se analizaron las evidencias de validez basadas en la estructura interna mediante análisis factorial confirmatorio con el estimador robusto WLSMV y matrices policóricas en el programa $\mathrm{R}$, poniendo a prueba tres modelos distintos, demostrando que el modelo oblicuo alcanzó índices de ajuste aceptables y mayor parsimonia: $\mathrm{CFI}=.929$, TLI=.920, RMSEA $=.083$ [.078-.088] y SRMR = .061 (Kline, 2005; Lacobucci, 2010; Manzano, 2017), confirmándose la pertinencia del modelo sistémico cognoscitivista para la prueba de 21 ítems, con siete ítems por cada uno de los tres factores correlacionados, propuesto por Barraza (2018).

También, se analizaron las evidencias de fiabilidad por el método de consistencia interna, encontrando magnitudes del coeficiente omega $>.80$ para las tres escalas con 7 ítems cada una, lo que se considera excelente (Carmines y Zeller,1979; Cascaes da Silva et al. 2015), y confirma que el SISCO SV-21 tiene un alto nivel de confiabilidad. Resultados similares se exponen en la investigación inicial realizada por Barraza (2018), aunque con el coeficiente alfa.

No obstante, entre las limitaciones de esta investigación se encuentran el tipo de muestreo, pues si bien la cantidad de observaciones es considerada como aceptable para validar un test (Comrey y Lee, 1992; Anthoine, Moret, Regnault, Sbille, y Hardouin, 2014), todos los participantes pertenecen al mismo nivel socioeconómico, y se ubican en Lima Metropolitana, por lo que futuras investigaciones deberían procurar ampliar y diversificar la composición de la muestra para generalizar los resultados y disminuir probables sesgos de medida. Otra de las limitaciones es su carácter transversal, ya que tampoco fue posible evaluar la estabilidad temporal de las puntuaciones del SISCO SV-21 mediante test-retest, por lo que en posteriores estudios se tendría que proponer por lo menos dos observaciones.

En síntesis, los datos permiten señalar que el Inventario SISCO SV-21 reúne evidencias psicométricas preliminares de validez y confiabilidad y equidad para su aplicación en estudiantes universitarios limeños. Aunque queda pendiente efectuar el análisis de invarianza factorial según sexo y edad, para determinar la posible equivalencia del inventario SISCO SV-21, lo que justificaría su adecuado uso en estudios comparativos y de prevalencia (Caycho-Rodríguez, 2017, Dominguez-Lara, 2016). Esto permitirá planificar mejor la aplicación de programas de intervención psicoeducativa con la finalidad de reducir los niveles de estrés. Finalmente, se deriva al lector a las referencias con las fuentes utilizadas en esta investigación.

\section{Referencias}

Alarcón, R. (2013). Métodos y diseños de investigación del comportamiento. (2. ${ }^{\mathrm{a}}$ ed.). Lima: Ricardo Palma.

Álvarez, S., Gallegos, L., \& Herrera, P., (2018). Estrés en estudiantes de Tecnología Superior. Universitas: Revista de Ciencias Sociales y Humanas, 28, 193-209.

American Psychiatric Association, (2010). Principios de ética en psicólogos y código de conducta. http://www.bibliopsi.org/docs/carreras/obligatorias/CFP/etica/farina/American\%20Psych ological\%20Association $\% 20 \% 20$ Principios $\% 20$ eticos $\% 20 \mathrm{de} \% 201$ os $\% 20$ psicologos $\% 20 \mathrm{y}$ $\% 20$ codigo $\% 20 \mathrm{de} \% 20$ conducta.pdf

Ancajima, L. (2017). Propiedades psicométricas del Inventario SISCO del Estrés Académico en universitarios de la ciudad de Trujillo (Tesis de pregrado). Universidad César Vallejo, 
Trujillo, Perú. Repositorio institucional UCV: http://repositorio.ucv.edu.pe/handle/20.500.12692/650

Ato, M., López, J., \& Benavente, A. (2013). Sistema de clasificación en diseños de investigación en psicología. Revista Anales de Psicología, 29(3), 1038-1059

Barraza, A. (2006). Un modelo conceptual para el estudio de estrés académico. Revista $\begin{array}{lllll}\text { Electrónica de Psicología } & \text { Iztacala, } & 9(3), & 110-129 .\end{array}$ $\mathrm{http} / /$ www.iztacala.unam.mx/carreras/psicologia/psiclin/principal.html

Barraza, A. (2007a). Estrés académico: un estado de la cuestión. Revista Electrónica Psicología Científica. http://www.psicologiacientifica.com/bv/psicologia-232-1-estres-academicoun-estado-de-lacuestion.html

Barraza, A. (2007b). Inventario SISCO del estrés académico. México: Universidad Pedagógica de Durango, Investigación Educativa.

Barraza, A. (2018). Inventario Sistémico Cognoscitivista SV-21 para el estudio del estrés académico. Segunda $\quad$ versión de $21 \quad$ ítems. http://www.ecorfan.org/libros/Inventario_SISCO_SV21/Inventario_sist\%C3\%A9mico_c ognoscitivista_para_el_estudio_del_estr\% $\%$ C3\%A 9 s.pdf

Barrera, A., Neira, M., Raipán, P., Riquelme, P., \& Escobar, B. (2019). Apoyo social percibido y factores sociodemográficos en relación con los síntomas de ansiedad, depresión y estrés en universitarios chilenos. Revista de Psicopatología y Psicología Clínica, 24(2), 105-115.

Berrío, N., \& Mazo, R. (2011). Estrés Académico. Revista de Psicología Universidad de Antioquia, 3(2), 65-82

Bertalanffy, L. (1976). Teoría General de los Sistemas. México: Fondo de Cultura Económica.

Brown, T. (2015). Confirmatory Factor Analysis for Apllied Research (2. ${ }^{\mathrm{a}}$ ed.). Nueva York, Estados Unidos, The Guilford Press.

Campo, A., \& Oviedo, H. (2008). Propiedades psicométricas de una escala: La consistencia interna. Revista Salud Pública, 10 (5), 831-839.

Carmines, E., \& Zeller, R. (1979). Reliability and Validity Assesment. California, Estados Unidos, Sage Publications

Cascaes da Silva, F., Goncalves, E., Valdivia, B., Graziele, G., Da Silva, T., Soleman, S., \& Da Silva, R. (2015). Estimadores de consistencia interna en las investigaciones en salud: el uso del coeficiente alfa. Revista Peruana de Medicina Experimental y Salud Pública, 32 (1), 129-138.

Castillo I., Barrios, A., \& Alvis, L. (2018). Estrés académico en estudiantes de enfermería de Cartagena, Colombia. Investigación en Enfermería: Imagen Y Desarrollo, 20(2), 1-11. https://www.redalyc.org/articulo.oa?id=145256681002

Caycho-Rodríguez, T. (2017). Intervalos de Confianza para el coeficiente alfa de Cronbach: aportes a la investigación pediátrica. Acta Pediátrica de México, 38(4), 291-294.

Colle, R. (2002). Teoría cognitiva sistémica de la comunicación. Santiago, Chile

Comrey, A., \& Lee, H. (1992). A first course in factor analysis (2. ${ }^{a}$ ed.). Nueva York: Psychology Press

Cortada de Kohan, N. (2002). Importancia de la investigación psicométrica. Revista Latinoamericana de Psicología, 229-240. https://www.redalyc.org/articulo.oa?id=805/80534303

Dominguez-Lara, S. A. (2016). Comparación del autoconcepto entre grupos, ¿sesgo o diferencias?: comentarios a Castillo et al. Revista Chilena de Pediatría, 87, 436. https://doi.org/10.1016/j.rchipe.2016.03.003

Hogan, T. (2004). Pruebas psicológicas. Una introducción práctica. México D.F., México: Manual Moderno.

Jerez, M., \& Oyarzo, C. (2015). Estrés Académico en estudiantes del Departamento de Salud de la Universidad de los Lagoa Osorno. Revista chilena de neuro-psiquiatría, 53(3), 149-157.

Kline, R. (2005). Principles and practice of structural equation modeling (2. ${ }^{\mathrm{a}}$ ed.). New York: Guilford

Lacobucci, D. (2010). Structural equations modeling: Fit indices, sample size, and advanced topics. Journal of Consumer Psychology, 20(1), 90-98. http://dx.doi.org/10.1016/j.jcps.2009.09.003 
Lazarus, R. (1966). Psychological stress and the coping process. Nueva York: McGraw-Hill.

Li, C. H. (2016). Confirmatory factor analysis with ordinal data: Comparing robust maximum likelihood and diagonally weighted least squares. Behavior research methods, 48(3), 936949. https://doi.org/10.3758/s1342

McDonald, R. P. (1999). Test theory: A unified treatment. Mahwah, NJ: Lawrence Erlbaum Associates, Inc.

Manzano, A. (2017). Introducción a los modelos de ecuaciones estructurales. http://www.scielo.org.mx/pdf/iem/v7n25/2007-5057-iem-7-25-67.pdf

Ministerio de Educación del Perú. (2019). Lineamientos para el cuidado integral de la salud mental en las universidades. https://www.gob.pe/institucion/minedu/noticias/52741minedu-y-el-minsa trabajan-con-21-universidades-publicas-en-el-cuidado-de-la-saludmental

Montero, I., \& León, O. (2002). Clasificación y descripción de las metodologías de investigación en psicología. Revista Internacional de Psicología y de la Salud, 2 (3), 503-508

Organización de las Naciones Unidas para la Educación, la Ciencia y la Cultura. (2020). COVID19 y educación superior: De los efectos inmediatos al día después. Análisis de impactos, respuestas políticas y recomendaciones. http://www.iesalc.unesco.org/wpcontent/uploads/2020/05/COVID-19ES130520.pdf

Orlandini, A. (1996). El estrés: qué es y cómo evitarlo. México: Fondo Cultura Económica

Peters, GJY. (2014). The alpha and the omega of scale reliability and validity: why and how to abandon Cronbach's alpha and the route towards more comprehensive assessment of scale quality. Europ Health Psychol, 16(2), 56-69. https://doi.org/10.31234/osf.io/h47fv

R Development Core Team, 2007. R: a language and environment for statistical compu-ting. R Foundation for Statistical Computing, Vienna, Austria, ISBN 3-900051-07-0, UR L htt $\mathrm{p}: / / \mathrm{w}$ w w.R-project.org

Román, C., Ortiz, F., \& Hernández, Y. (2008). El estrés académico en estudiantes latinoamericanos de la carrera de Medicina. Revista Iberoamericana de Educación, 46(7), $1-8$

Rossi, R. (2012). Los consejos del psicólogo para superar el estrés. Bacerlona: De Vecchi Ediciones

Rosseel, Y., Oberski, D., Byrnes, J., Vanbrabant, L., Savalei, V., Merkle, E., \& Jorgensen, D. (2018). Package 'lavaan' 0.6-3. https://cran.r-project.org/web/packages/lavaan/lavaan.pdf

Salud mental: El 44\% de los estudiantes universitarios ha estado con tratamiento psicológico (29 de abril del 2019). CNN CHILE. https://www.cnnchile.com/pais/mas-de-500-ninos-yvadolescentes-viven-en-situacion-de-calle-en-chile 20190506/

Selye, H. (1936). Thymus and Adrenals in the Response of the Organism to Injuries and Intoxications. The british Journal of Experimental Pathology, 17(3), 234-248

Selye, H. (1973). The Evolution of the Stress Concept: The originator of the concept traces its development from the discovery in 1936 of the alarm reactions to modern therapeutic applications of syntoxic and catatoxic hormones. American Scientist, 61(6), 692-699 